Methods Retrospective case note review of $100 \mathrm{MSU}$ requests at a sexual health clinic between 2014 and 2015. The associated clinical presentations and culture results were identified.

Results 14\% of MSU were requested within guidelines. 29\% (4/ $14)$ of those were positive, compared to $22 \%$ (19/86) not requested within guidelines. Indications outside guidelines associated with positive culture included: women with lower urinary tract symptoms (11), men with dysuria only (3), pelvic inflammatory disease (2), asymptomatic with positive urine dipstick (2), and vaginal discharge (1). 15/23 were sensitive and 8/23 were resistant to trimethoprim.

Discussion/conclusion MSU is often requested inappropriately. This generates positive results associated with clinical presentations unlikely to indicate UTI. Greater awareness amongst clinicians of appropriate indications for MSU will support optimal resource utilisation in sexual health clinics. Resistance to our first line antibiotic, trimethoprim, was identified. Resistance patterns should be monitored so clinicians can confidently prescribe empirical treatment for lower UTI in nonpregnant women.

\section{P029 HIS-UK CONDOM STUDY: AN INTERVENTION DEVELOPMENT STUDY WORKING WITH YOUNG MEN TO IMPROVE CONDOM USE SKILLS AND ENHANCE CONDOM USE EXPERIENCES}

${ }^{1}$ Cynthia Graham, ${ }^{1}$ Sydney Anstee*, ${ }^{1}$ Nicole Stone, ${ }^{2}$ Katherine Brown, ${ }^{2}$ Katie Newby, ${ }^{1}$ Roger Ingham. ${ }^{1}$ University of Southampton, Southampton, UK; ${ }^{2}$ Coventry University, Coventry, UK

\subsection{6/sextrans-2016-052718.83}

Background The Kinsey Institute ${ }^{\circledR}$ Homework Intervention Strategy (KIHIS), designed to improve condom skills, enjoyment and self-efficacy, has demonstrated early evidence of efficacy in U.S. studies. The KIHIS places the impetus for change on the individual through solitary practice: experimenting with different condoms/lubricants; identifying best 'fit $\&$ feel'; and focusing on physical sensations.

Aim(s)/objectives To identify behaviour change techniques (BCTs) in KIHIS; to adapt and develop KIHIS for the UK context; to manualise and evaluate HIS-UK.

Methods Literature synthesis to identify additional BCT components and methods of delivery to address condom fit and feel. Stakeholder and user consultation through qualitative interviews ( $\mathrm{n}=15$ men aged 16-25); advisory groups (e.g. consultants, commissioners); workshops (e.g. health promotion professionals)

Results Searches of online databases, July 2015, identified 1044 condom use intervention studies published since 2006; of these, 123 studies tested the effectiveness of behavioural interventions on condom use in high income countries - and only five targeted 'fit \& feel' issues. In total 22 BCTs were identified, 16 of which were selected for inclusion in HIS-UK. Consultations have demonstrated enthusiasm for this 'fit \& feel' approach, have enabled us to gauge UK preferences (e.g. condom kit contents) and have informed adaptation of the intervention.

Discussion This work ensures that the targeted outcomes, behaviour determinants and proposed mechanisms of action for HISUK are specified, so that future conclusions can be drawn about what works and why. An adapted and manualised intervention is currently being piloted for viability and operability among 50 men aged $16-25$ years.

\section{P030 DEVELOPMENT, IMPLEMENTATION AND EARLY EVALUATION OF A PILOT CERVICAL SCREENING CLINIC FOR WOMEN WHO HAVE BEEN SEXUALLY ASSAULTED}

${ }^{1}$ Jill Zelin*, ${ }^{2}$ Louise Cadman, ${ }^{1}$ Jane Vosper, ${ }^{3,1}$ Pavan Amara. ${ }^{1}$ Barts Sexual Health Centre, St. bartholomew's Hospital, Barts Health, London, UK; ${ }^{2}$ Centre for Cancer Prevention, Wolfson Institute of Preventive Medicine, Queen Mary University of London, London, UK; ${ }^{3}$ My Body Back Project, London, UK

\subsection{6/sextrans-2016-052718.84}

Background One in five women does not attend for cervical screening when invited. This includes those who have experienced sexual violence, putting them at increased risk of cervical cancer. A pilot clinic was set up in partnership with the My Body Back Project (MBB). MBB supports women who have been raped to regain confidence and control of their body and health. The clinic offers cervical screening and STI testing for these women with a multidisciplinary collaboration between doctor, nurse, psychologist and $\mathrm{MBB}$ advocate as facilitator. It aims to provide time, space, shared control and understanding of the particular difficulties faced.

Aim To evaluate acceptability and uptake of a pilot cervical screening clinic for women with a history of sexual assault.

Methods Questionnaires were collected from women attending between August and December 2015.

Results 30 women attended (median age 34.4 years). $48.3 \%$ had never been screened and $72.4 \%$ were significantly overdue.

\begin{tabular}{|c|c|c|c|}
\hline Cytology result & $\begin{array}{l}\text { Cytology at visit } 1 \\
\left(\mathrm{~N}=26^{*}\right)\end{array}$ & $\begin{array}{l}\text { Cytology at visit } 2 \\
(\mathrm{~N}=3)\end{array}$ & $\begin{array}{l}\text { Total (\%) } \\
\text { smears taken }\end{array}$ \\
\hline Negative & 21 & 2 & $23(79.3)$ \\
\hline Unsatisfactory & 1 & 0 & 1 (3.4) \\
\hline Borderline HPV+ & 1 & 0 & $1(3.4)$ \\
\hline Results pending & 3 & $1^{* *}$ & $4.13 .8)$ \\
\hline $\begin{array}{l}\text { No cytology } \\
\text { taken }\end{array}$ & $2^{* * *}$ & 0 & $x$ \\
\hline TOTAL & 26 & 3 & $29(100)$ \\
\hline
\end{tabular}

Feedback showed $96.7 \%$ of women found the clinic very useful, the advocate helpful and felt understood. $86.2 \%$ found the smear taker and psychologist together helpful and 100\% would recommend the service. Confidence in their ability to have a smear increased from slightly/in some situations before their examination to in some/most situations afterwards. Common qualitative themes included not feeling rushed, feeling in control and having needs understood.

Discussion/conclusion The uptake, waiting list and feedback from women suggest that this is a necessary and appreciated clinic. Further evaluation is required in order to improve and sustain the service.

\section{P031 ESTIMATING LOCAL CHLAMYDIA INCIDENCE AND PREVALENCE USING SURVEILLANCE DATA}

1,2Joanna Lewis* ${ }^{1,2}$ Peter White. 'Department of Infectious Disease Epidemiology, Imperial College London, London, UK; ${ }^{2}$ Modelling and Economics Unit, Public Health England, London, UK

10.1136/sextrans-2016-052718.85 
Background/introduction Understanding patterns of chlamydia prevalence is important for addressing inequalities and assessing interventions. Population-based surveys are expensive; the best UK data come from the Natsal national surveys which are only available once per decade and not powered to compare prevalence in different localities. Estimates at finer spatial and temporal scales are required.

Aim(s)/objectives We aimed to estimate chlamydia prevalence from numbers of tests and diagnoses reported in surveillance data.

Methods Our method is based on a simple model for the infection, testing and treatment processes and informed by the literature on infection natural history and treatment seeking behaviour. By combining this information with surveillance data we obtain estimates of chlamydia screening rates, incidence and prevalence. We validate and illustrate the method by application to national and local-level data from England.

Results Estimates of national prevalence by sex and age group agree with results from the Natsal-3 survey. They could be improved by additional information on the number of diagnoses that were symptomatic. There is substantial local-level variation in prevalence, with more infection in deprived areas. Incidence in each sex is strongly correlated with prevalence in the other. Importantly, we find that positivity (the proportion of tests which were positive) does not provide a reliable proxy for prevalence.

Discussion/conclusion This approach provides a powerful tool to identify prevalence trends with time and location, and understand the effects of control strategies. Estimates could be more accurate if surveillance systems recorded which patients were symptomatic and the duration of symptoms before care-seeking.

\section{P032 DEVELOPING A SECTOR LED IMPROVEMENT APPROACH TO SEXUAL HEALTH}

Dianne Draper. Public Health England, London, UK

10.1136/sextrans-2016-052718.86

Background Sector-led improvement (SLI) is an approach where local authorities (LA) help each other to continuously improve. It is replacing more traditional performance frameworks, however, the approach lacks a clear methodology. We developed and piloted an evidence-based SLI approach to drive improvements in sexual health $(\mathrm{SH})$ within LAs.

Aim(s) To develop and pilot an evidenced-based SLI toolkit

Methods Key components for effective SLI were identified following a review of the published literature. These were embedded within a co-produced, peer-review toolkit which was piloted by $\mathrm{SH}$ commissioners and key stakeholders from four local authorities. The pilot focussed on delivery of local chlamydia screening programmes.

Results Several key clinical and structural issues were identified through the SLI approach including low coverage, the potential to improve partner notification outcomes, low re-testing rates, threats from a reduction in spend and unclear governance. These have been put into a local action plan to focus and drive quality improvement activities. The impact of the action plans will be the focus of a follow up meeting planned for six months after the final peer review meeting involving wider stakeholders.

Discussion The SLI toolkit offers a systematic approach to evaluating complex programme activities. It was well received locally and helped key stakeholders to gain insight, catalyse self- reflection and prioritise areas for change in order to drive quality and improvement.

\section{P033 SAFETY FIRST: COMBINED ORAL CONTRACEPTIVE PRESCRIBING IN PRIMARY CARE}

Sophie Russell. University Hospitals Bristol NHS Foundation Trust, Bristol, UK

10.1136/sextrans-2016-052718.87

Background/introduction $80 \%$ of contraceptive care occurs in the general practice (GP) setting. UK Medical Eligibility Criteria (UKMEC) provides clear guidelines for the safe provision of appropriate contraception. The Faculty of Sexual and Reproductive Health (FSRH) and the National Institute of Clinical Excellence (NICE) offer further recommendations for combined oral contraceptive pill (COCP) initiation and continuation.

Aim(s)/objectives To establish if primary care COCP prescribing was compliant with national safety and best practice guidelines.

Methods The EMIS database of an average size, inner city GP surgery was used to analyse COCP consultations between 11/10/ 2015 and 11/01/2016.

Results 56 women aged 14-39 years were prescribed the COCP. In $41 \%$ of consultations there was substandard documentation of medical eligibility.

\begin{tabular}{|c|c|}
\hline UKMEC Condition & $\%$ Consultations Lacking Documentation \\
\hline Venous thromboembolism & $28 \%$ \\
\hline Smoking status & $25 \%$ \\
\hline Blood pressure & $16 \%$ \\
\hline Body mass index & $16 \%$ \\
\hline \multicolumn{2}{|c|}{$\begin{array}{l}\text { The COCP was prescribed without specialist input for three patients with a UKMEC } 3 \text { con- } \\
\text { dition: systolic blood pressure } 143 \text {, undiagnosed breast lump and first degree family history } \\
\text { of venous thromboembolism. } 87 \% \text { patients did not receive advice about missed pill rules; } \\
\text { and } 21 \% \text { of eligible women were not advised on the benefits of long acting reversible con- } \\
\text { traception (LARC). Only } 8 \% \text { of patients were risk assessed for sexually transmitted infec- } \\
\text { tions (STIs) and no women were offered HIV screening. }\end{array}$} \\
\hline
\end{tabular}

Discussion/conclusion The safety of COCP prescribing could be enhanced by improved application of UKMEC criteria. Promotion of safe sex was not undertaken despite high incidence of STIs and local availability of LARC options.

\section{P034 ACCESS TO SEXUAL AND REPRODUCTIVE HEALTH IN THE CALAIS "JUNGLE CAMP" FOR WOMEN. A VOLUNTEER PERSPECTIVE}

\footnotetext{
${ }^{1,2}{ }^{2}$ Fionnuala Finnerty*, ${ }^{1}$ Aliza Amlani, ${ }^{2}$ Elaine Ortiz, ${ }^{1,3}$ Daniel Richardson. ${ }^{1}$ Brighton \& Sussex County Hospital, Brighton, UK; ${ }^{2}$ Hummingbird Project, Brighton, UK; ${ }^{3}$ Brighton \& Sussex Medical School, Brighton, UK
}

\subsection{6/sextrans-2016-052718.88}

Background/introduction The number of women in the "jungle" camp in Calais, France increased in 2015 but definitive numbers are unknown. Health services report these women are a difficult to access population. Multiple small groups of grassroots volunteers support initiatives in the camp and have access to vulnerable groups.

Aims/objectives To survey volunteers opinions on access to sexual and reproductive health (SRH) care in the "jungle" camp 